\title{
Beyond-the-Standard Model Higgs Physics using the ATLAS Experiment
}

\author{
Loan Truong ${ }^{1,2,3 a}$ on behalf of the ATLAS Collaboration \\ ${ }^{1}$ INFN Gruppo Collegato di Udine, Sezione di Trieste, Udine, Italy \\ ${ }^{2}$ ICTP, Trieste, Italy \\ ${ }^{3}$ SISSA, Trieste, Italy
}

\begin{abstract}
Recent searches for the Higgs boson in the context of beyond the Standard Model performed by the ATLAS experiment are presented: high mass Higgs boson searches, lepton flavour violating Higgs decay, NMSSM, constraint from the search for three photons. The interpretation based on the measurements of Higgs couplings is shown, along with the constraint on the Higgs boson invisible decays. The search for invisible decays of a Higgs boson produced in association with a $Z$ boson was performed with both $\sqrt{s}=7$ and $8 \mathrm{TeV}$ while the rest were performed using the $\sqrt{s}=8 \mathrm{TeV}$ data of proton-proton collisions collected by the ATLAS experiment. No significant excess of data over the predicted background is observed and limits are placed in certain quantities depending on the searches.
\end{abstract}

\section{Introduction}

The ATLAS experiment at the LHC discovered the Higgs boson in July 2012. So far, there have been many measurements on its couplings and mass [1-5], the results are consistent with the Standard Model (SM) predictions. Nevertheless, these measurements have not yet excluded a large range of other extensions of the SM.

Exploring whether there are additional Higgs bosons or exotic decay of the SM Higgs boson can give us direct evidences about physics beyond the Standard Model (BSM). Those include the two Higgs doublet models (2HDMs) [6-9], next-to-minimal superymmetric SM (NMSSM) [10, 11], composite Higgs models [12, 13], which favour the existence of other Higgs bosons in the high-mass regime, as well as lepton flavour violating (LFV) Higgs decay, three photons Higgs decay. There are strong evidences of dark matter from astrophysical observations which could be explained by the existence of weakly interacting massive particles (WIMPs, see Ref. [14] and the references therein). The observed Higgs boson might decay to dark matter or other stable or long-lived particles which do not interact significantly with a detector [15-19] leading to Higgs boson invisible decay.

The report is organized as follows: Sec. 2 describes the high mass-Higgs boson searches, Sec. 3 describes the LFV Higgs decay search, Sec. 4 describes the search for Higgs bosons

a e-mail: lotruong@cern.ch 
in the context of NMSSM, Sec. 5 describes the search for a Higgs boson decaying to three photons or more, Sec. 6 summarizes some constraints on BSM models using Higgs boson couplings and mass measurements, Sec. 7 describes the combination of the searches for invisible decays of the Higgs boson. Finally concluding remarks are in Sec. 8. Details about the ATLAS detector can be found in Ref. [20].

\section{High masses searches}

Two searches for a high-mass Higgs boson $\mathrm{H}$ were performed in the $H \rightarrow W W \rightarrow \ell \nu \ell \nu, \ell \nu q q[21]$ and $H \rightarrow Z Z \rightarrow \ell \ell \ell \ell, \ell \ell \nu \nu, \ell \ell q q, \nu \nu q q[22]$ channels. No significant deviations were observed. The results were interpreted separately for $\mathrm{VBF}, \mathrm{ggF}$ production modes as upper limits at 95\% CL (see Fig. 1 and Tab. 1) on $\sigma_{H} \times B R(H \rightarrow W W / Z Z)$ for heavy Higgs boson which has a narrow width.

Table 1: Upper limits on the production cross section of the high mass-Higgs boson in different weak vector boson decay modes [21, 22].

\begin{tabular}{c|c|c|c}
\hline \multirow{2}{*}{ Limits on } & \multirow{2}{*}{$m_{H}[\mathrm{GeV}]$} & Upper Limit $[\mathrm{fb}]$ for $\mathrm{H}$ production mode \\
\cline { 3 - 4 } & & $\mathrm{ggF}$ & VBF \\
\hline$\sigma_{H} \times B R(H \rightarrow W W)$ & 1500 & 21 & 6 \\
$\sigma_{H} \times B R(H \rightarrow Z Z)$ & $195 \div 950$ & $530 \div 8$ & $310 \div 9$ \\
\hline
\end{tabular}

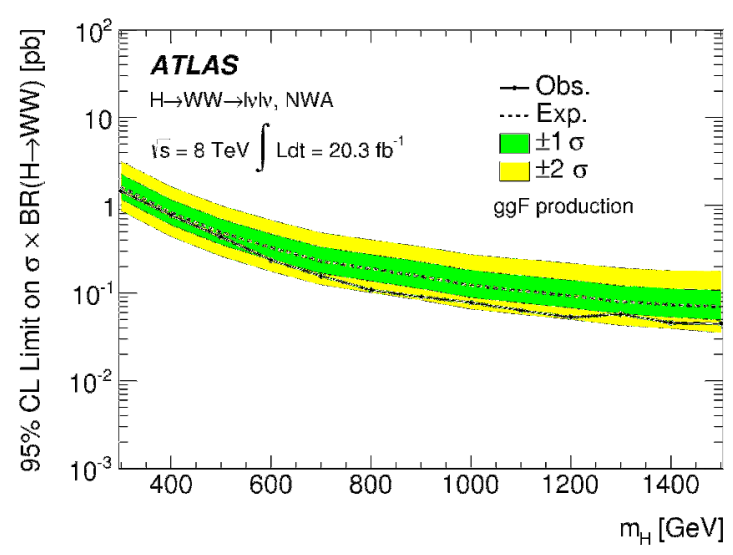

(a) $H \rightarrow W W \rightarrow \ell \nu \ell \nu$

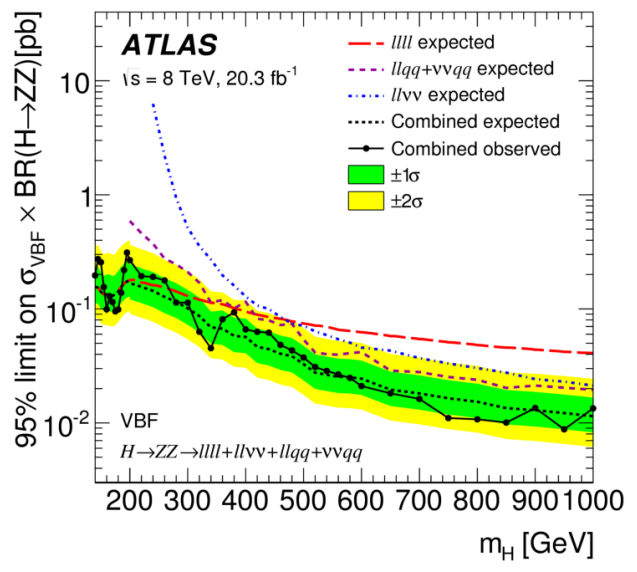

(b) $H \rightarrow Z Z \rightarrow \ell \ell \ell \ell, \ell \ell \nu \nu, \ell \ell q q, \nu \nu q q$

Figure 1: Limits set on the production cross section of the high mass-Higgs boson in (a) $W W[21]$, (b) $Z Z[22]$ resonance searches.

\section{Lepton flavour violating decays}

The LFV Higgs decays $(H \rightarrow \tau \mu, \tau e, \mu e)$ arise at tree level based on assumed flavour violating Yukawa interactions [23]. A search for LFV Higgs decays was done in the channel $H \rightarrow \mu \tau_{\text {had }}$ 
in hadronic $\tau$ decays [24]. A fit to the reconstructed mass distribution in data was performed (see Fig. 2) resulting in the best fit of $B R(H \rightarrow \mu \tau)=0.77 \pm 0.62 \%$, upper limit on $B R(H \rightarrow$ $\mu \tau)$ at $95 \%$ CL: $1.85 \%(1.24 \%)$ obs( $\exp )$.

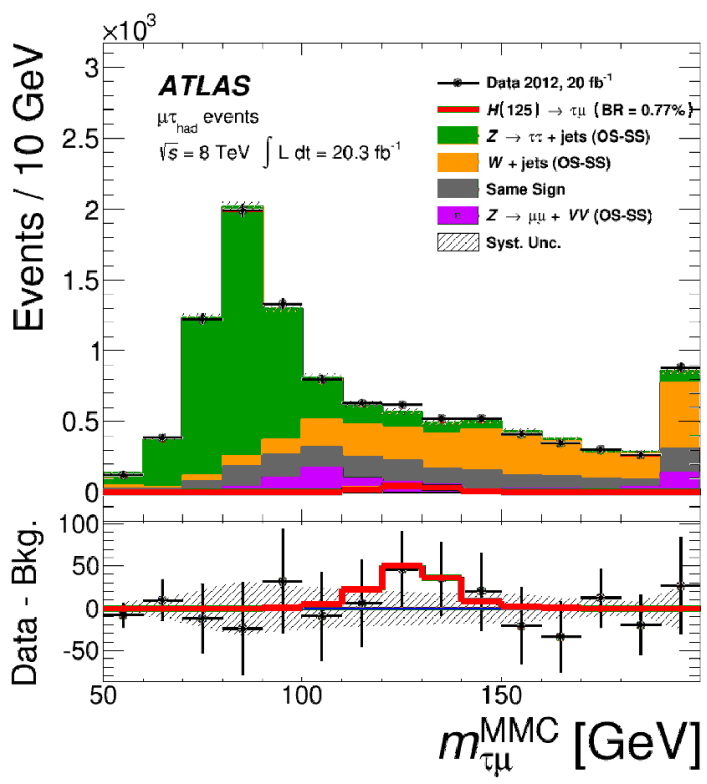

Figure 2. The reconstructed mass of the system of observed muon, $\tau$ (hadronic decay products) \& $E_{\mathrm{T}}^{\text {miss }}$ objects [24] by means of the Missing Mass Calculator distribution.

\section{NMSSM}

The NMSSM contains an additional pseudoscalar Higgs boson $(a)$, generally assumed to have a mass lower than the observed Higgs boson (h) since its mass is protected by a Peccei-Quinn symmetry. A search for the decay to a pair of the lightest neutral pseudoscalar Higgs $a$ of either the $125 \mathrm{GeV}$ Higgs (h) or a second CP-even Higgs (H) was performed [25], where one $a$ boson decays to $2 \mu$ and the other decays to $2 \tau$. As a result, the most stringent upper limit on the branching ratio of the $\mathrm{h}$ boson decaying to the non-SM particles was set at $3.5 \%$ for $m_{a}=3.75 \mathrm{GeV}$ (see Fig. 3).

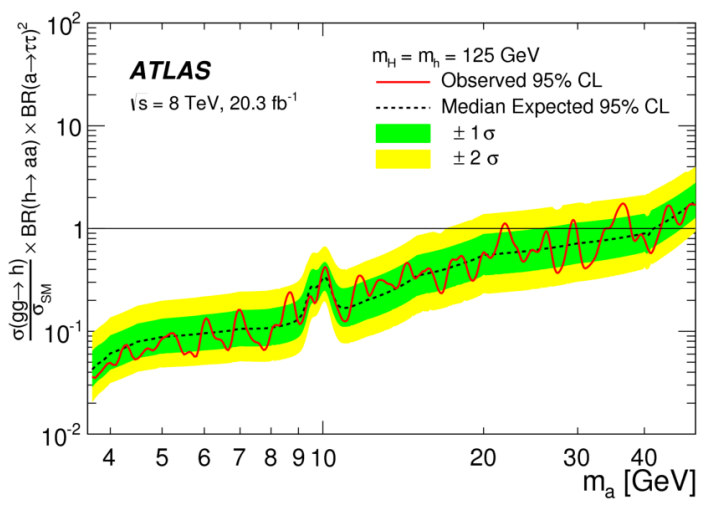

Figure 3. Limit for $\sigma_{g g H_{S M}} \times B R$ of h SM decays to aa $\times B R$ of a decays to $\tau \tau$ vs $m_{a}[25]$. 


\section{Search for 3 photons}

A search for events with at least $3 \gamma$ was done [26]. The model-independent interpretations are the first of their kind. One of the interpretations was performed for a SM Higgs boson (h) decaying to four photons via a pair of intermediate pseudoscalar particles $(a)$. Limits on the cross section times $B R(h \rightarrow a a) \times B R(a \rightarrow \gamma \gamma)^{2}$ was set to be $<10^{-3} \sigma_{S M}$ for $10 \mathrm{GeV}<$ $m_{a}<62 \mathrm{GeV}$ (see Fig. 4).
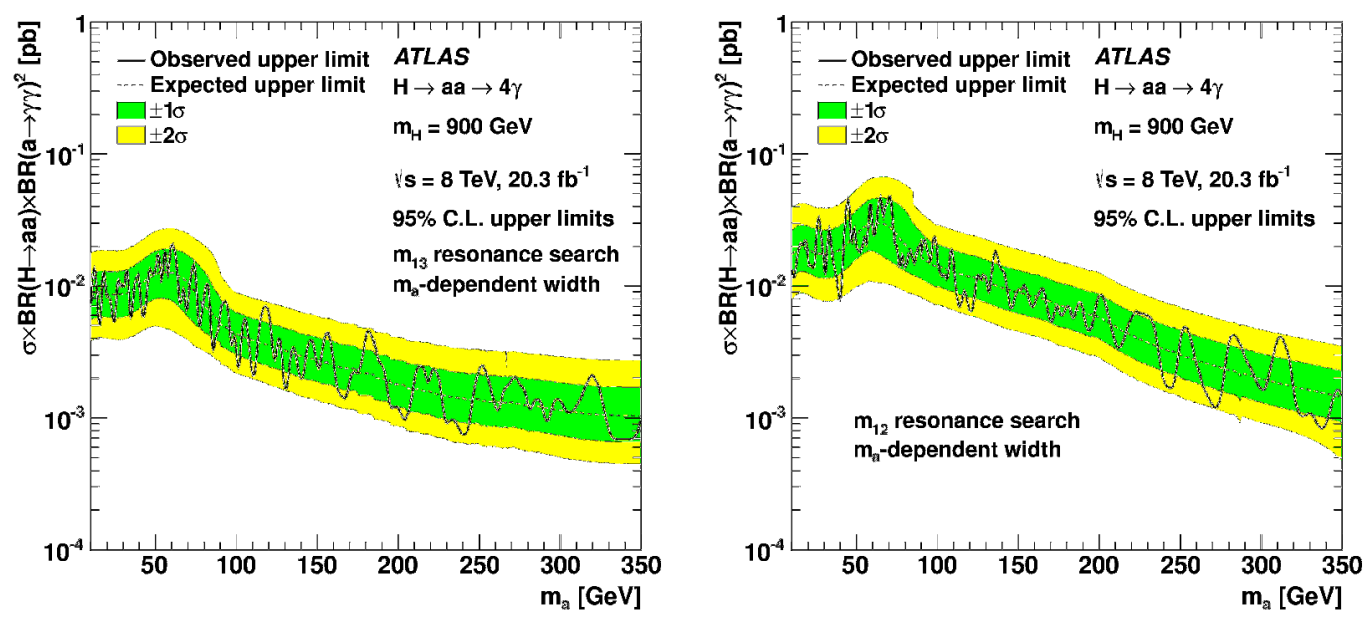

Figure 4: Left: For SM Higgs $h \rightarrow a a \rightarrow \gamma \gamma \gamma \gamma: \sigma \times B R(h \rightarrow a a) \times B R(a \rightarrow \gamma \gamma)^{2}<10^{-3} \times \sigma_{S M}$ for $10 \mathrm{GeV}<m_{a}<62 \mathrm{GeV}$. Right: for heavy Higgs boson-like scalar: $\sigma_{H} \times B R(H \rightarrow a a) \times$ $B R(a \rightarrow \gamma \gamma)^{2}<0.02$ to $0.001 \mathrm{pb}$ (depending upon $\left.m_{H}, m_{a}\right)[26]$.

\section{Beyond the Standard Model constraints via Higgs boson couplings}

BSM constraints [27] were performed using the measured production and decay rates of the Higgs boson $(\gamma \gamma, Z Z, W W, Z \gamma, b b, \tau \tau, \& \mu \mu ; t \bar{t} h$ with $h \rightarrow \gamma \gamma, b \bar{b} \&$ multileptons). The constraints include the probe on the scaling of the couplings with mass, setting limits on parameters in extensions of the SM such as composite Higgs boson, 2HDMs. They are described in details below.

\subsection{Mass scaling of couplings}

In this analysis, the observed rates in different channels were used in a fit to determine how the Higgs boson couplings to other particles scale with the masses of those particles [28]. Each coupling (with fermion $\mathrm{f}$ and boson $\mathrm{V}$ ) was scaled in terms of "vacuum expectation value (vev)" $M$ and mass scaling parameter $\epsilon$ (in the SM vev $v \approx 246 \mathrm{GeV}$ and $\epsilon \rightarrow 0$ ) as $\kappa_{f}=v \frac{m_{f}^{\epsilon}}{M^{1+\epsilon}}, \kappa_{V}=v \frac{m_{V}^{2 \epsilon}}{M^{1+2 \epsilon}}$, where $m$ is the mass of the particle. As a result of the fit, the best fit value is consistent within one standard deviation with the SM (see Fig. 5), observed values for $\epsilon$ and $M$ are shown in Tab. 2 . 
Table 2: Observed and expected measurements of the mass scaling parameter $\epsilon$ and the vev parameter $M[27]$.

\begin{tabular}{c|cc}
\hline Parameter & Obs. & Exp. \\
\hline$\epsilon$ & $0.018 \pm 0.039$ & $0.000 \pm 0.042$ \\
$M$ & $224_{-12}^{+14} \mathrm{GeV}$ & $246_{-16}^{+19} \mathrm{GeV}$ \\
\hline
\end{tabular}

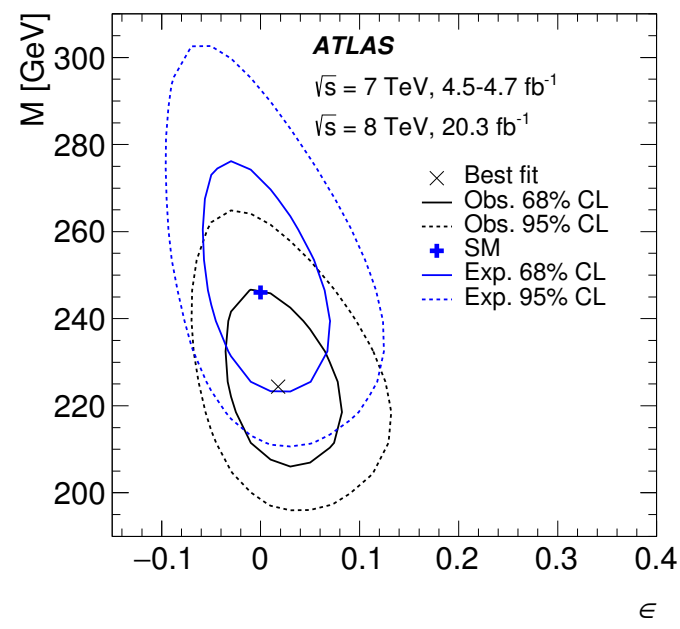

Figure 5. 2-D confidence intervals as a function of the mass scaling factor $\epsilon$ and the vev parameter $M[27]$.

\subsection{Minimal composite Higgs model}

Minimal Composite Higgs Models (MCHM) [12, 13] provides a possible explanation for the scalar naturalness problem. It suggests that the Higgs boson is a composite, pseudoNambu-Goldstone boson rather than an elementary particle. Higgs couplings are modified as functions of compositeness scale-f: $\xi=v^{2} / f^{2}$. There are different MCHM models with different modifications of the Higgs coupling such as MCHM4 [12] with $\sqrt{\kappa}=\kappa_{V}=\kappa_{F}=1-\xi$ and MCHM5 [13] with $\kappa_{V}=1-\xi, \kappa_{F}=\frac{1-2 \xi}{\sqrt{1-\xi}}$. The SM is recovered in the limit $\xi \rightarrow 0$, namely $f \rightarrow \infty$. A fit was performed in the parameter space of $\left[\kappa_{F}, \kappa_{V}\right]$ (see Fig. 6). As a result, limits at 95\% CL are obtained in the order of obs (exp), for MCHM4: f $>710$ (510) GeV, MCHM5: f > 780 (600) GeV.

\subsection{Two Higgs Doublet Models}

2HDMs models introduce two complex SU(2) doublets of scalar fields and there are 4 types of them (as summarized in Tab. 3). They predict five Higgs bosons: two neutral CP-even bosons $\mathrm{h}$ and $\mathrm{H}$, one neutral $\mathrm{CP}$-odd boson $\mathrm{A}$, and two charged bosons $\mathrm{H}^{ \pm}$. When considering the CPconserving case, there are 6 sensitive parameters in the analysis: 4 masses $m_{h}, m_{H}, m_{H^{ \pm}}, m_{A}$ $\& 2$ mixing angles $\alpha, \beta$. Where $\tan \beta=v_{1} / v_{2}$ is the ratio of the two Higgs doublets' vevs which satisfy $v_{1}^{2}+v_{2}^{2}=v^{2} \approx(246 \mathrm{GeV})^{2}, \alpha$ : mixing angle between $\mathrm{h} \& \mathrm{H}$. Assumptions for interpretations are that the $125 \mathrm{GeV}$ boson is the light Higgs, no radiative corrections from BSM for the production of Higgs boson, only SM decays. Gauge invariance requires the 


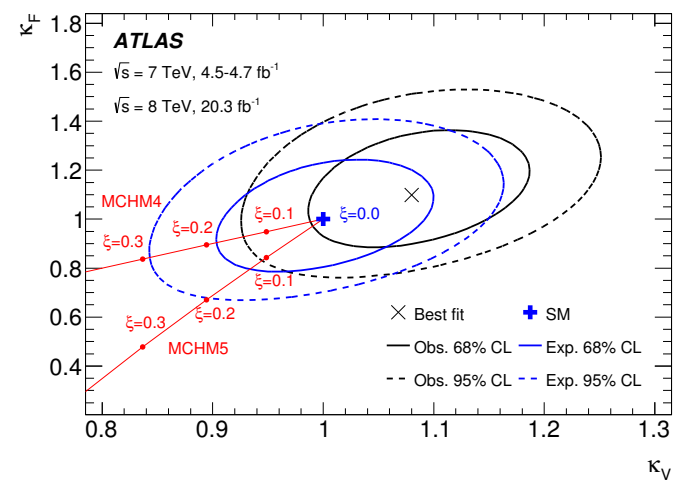

Figure 6. 2-D likelihood contours in the $\left(\kappa_{V}, \kappa_{F}\right)$ coupling scale factor plane [27].

Table 3: Couplings of the light Higgs boson $h$ to weak vector bosons $\left(\kappa_{V}\right)$, up-type quarks $\left(\kappa_{u}\right)$, down-type quarks $\left(\kappa_{d}\right)$, and charged leptons $\left(\kappa_{\ell}\right)$, expressed as ratios to the corresponding $\mathrm{SM}$ predictions in $2 \mathrm{HDM}$ of various types.

\begin{tabular}{c|cccc}
\hline Coupling scale factor & $\begin{array}{c}\text { Type I } \\
\text { (fermiophobic) }\end{array}$ & $\begin{array}{c}\text { Type II } \\
\text { (MSSM-like) }\end{array}$ & Lepton-specific & Flipped \\
\hline$\kappa_{V}$ & \multicolumn{4}{|c}{$\sin (\beta-\alpha)$} \\
\hline$\kappa_{u}$ & \multicolumn{4}{c}{$\cos (\alpha) / \sin (\beta)$} \\
\hline$\kappa_{d}$ & $\cos (\alpha) / \sin (\beta)$ & $-\sin (\alpha) / \cos (\beta)$ & $\cos (\alpha) / \sin (\beta)$ & $-\sin (\alpha) / \cos (\beta)$ \\
\hline$\kappa_{\ell}$ & $\cos (\alpha) / \sin (\beta)$ & $-\sin (\alpha) / \cos (\beta)$ & $-\sin (\alpha) / \cos (\beta)$ & $\cos (\alpha) / \sin (\beta)$ \\
\hline
\end{tabular}

couplings of the two neutral, CP-even Higgs bosons to vector bosons relative to their SM values to be:

$$
\begin{gathered}
g_{h V V}^{2 H D M} / g_{h V V}^{S M}=\sin (\beta-\alpha) \\
g_{H V V}^{2 H D M} / g_{H V V}^{S M}=\cos (\beta-\alpha)
\end{gathered}
$$

with the convention: $\sin (\beta-\alpha) \geq 0$. SM-like alignment limit is retrieved at $\cos (\beta-\alpha)=0$.

Figure 7 shows the regions of the $[\cos (\beta-\alpha), \tan \beta]$ plane that are excluded at $95 \%$ CL for each of the four types of $2 \mathrm{HDMs}$, overlaid with the exclusion limits expected for the SM Higgs sector. A physical boundary of $\kappa_{V} \leq 1$ is there in all four 2HDM types and restricts the profile likelihood ratio. The data are consistent with the alignment limit within approximately one standard deviation or better in each of the model.

\section{Higgs invisible decays}

Limits on the branching ratio of Higgs boson invisible decay [27] are set and subsequently used to constrain on a Higgs-portal dark matter model [29].

\subsection{The combination of Higgs invisible decay channels}

Direct searches for the SM Higgs boson h decaying into invisible particles used the following strategies: selecting events with large missing transverse momentum $\left(E_{\mathrm{T}}^{\mathrm{miss}}\right)$, using particles 


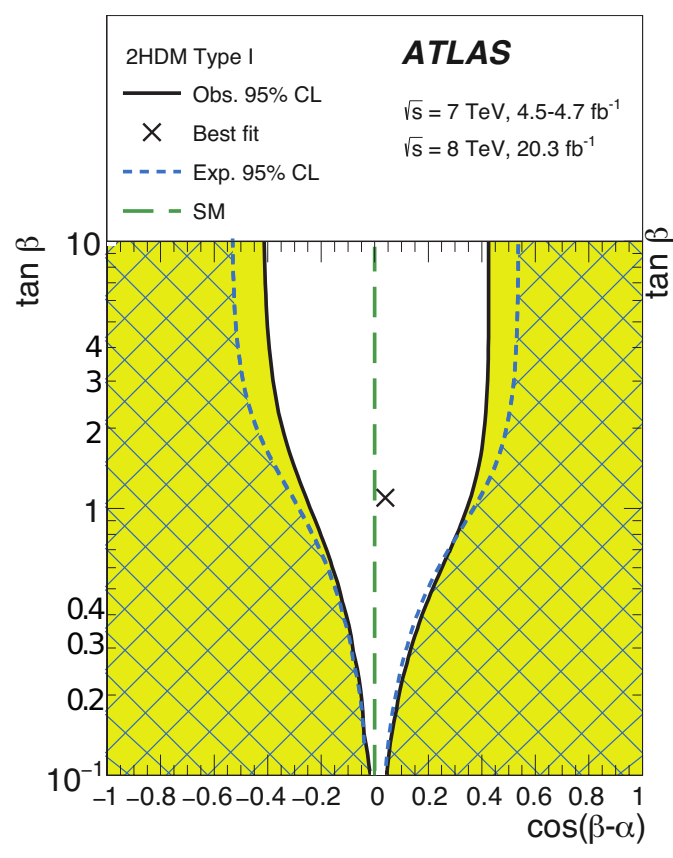

(a) Type I

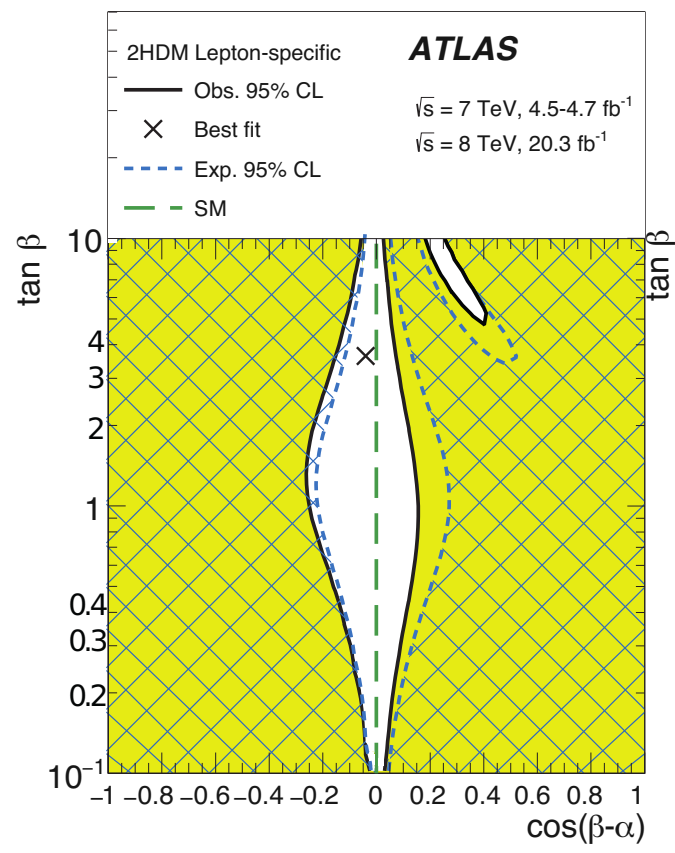

(c) Lepton-specific

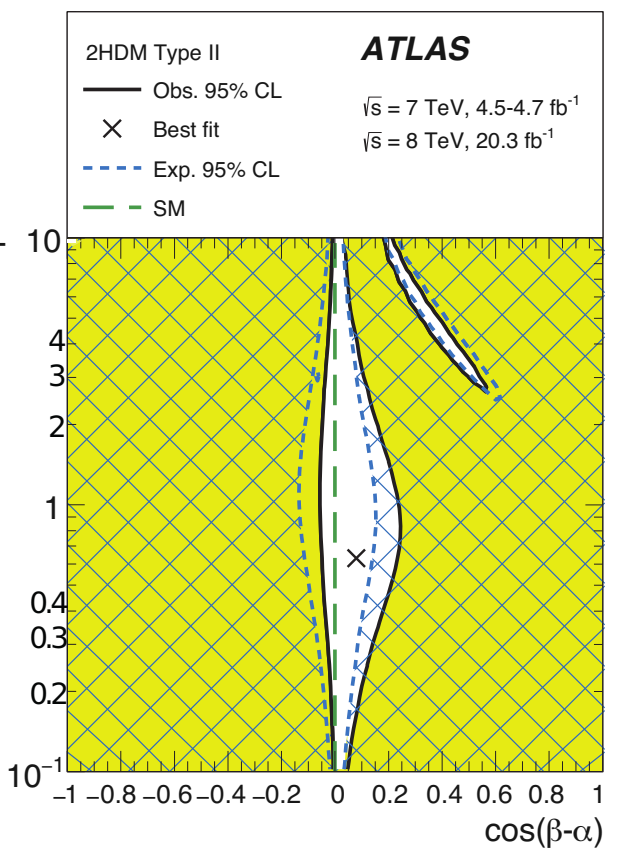

(b) Type II

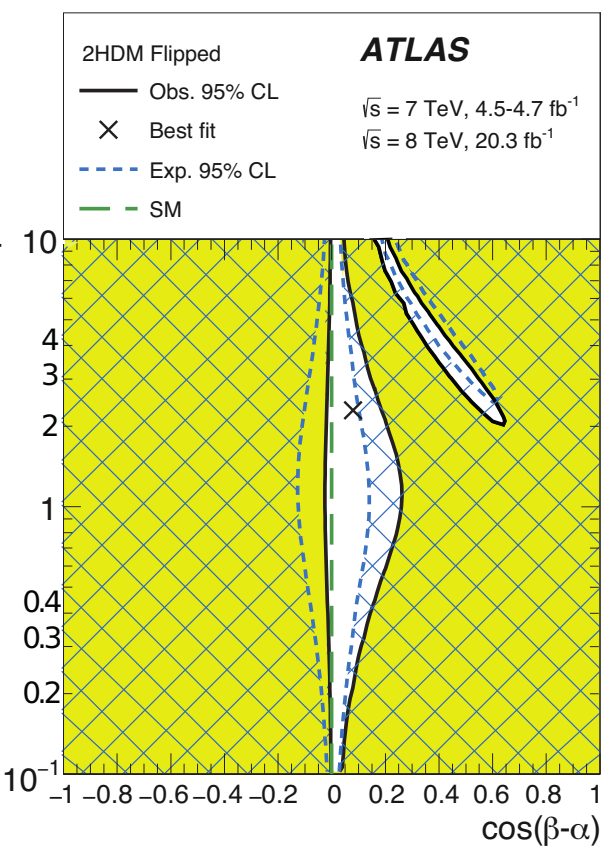

(d) Flipped

Figure 7: Regions of the $(\cos (\beta-\alpha), \tan \beta)$ plane of four types of 2 HDMs excluded by fits to the measured rates of Higgs boson production and decays. The likelihood contours where $-2 \ln \Lambda=6.0$, corresponding approximately to the $95 \%$ CL (2 std. dev.), are indicated for both the data and the expectation assuming the SM Higgs sector. The cross in each plot marks the observed best-fit value. The light shaded and hashed regions indicate the observed and expected exclusions, respectively. The plots are shown for $\sin (\beta-\alpha) \geq 0$ without loss of generality [27]. 
produced associated with the Higgs boson to tag the Higgs, assuming productions (and acceptance) as in the SM where $B R(h \rightarrow Z Z \rightarrow 4 \nu)=1.2 \times 10^{-3}$. The SM branching ratio is very little so any observation would be an indication of BSM physics. They include the analyses: $\mathrm{Z}(\rightarrow \mathrm{ll}) \mathrm{h} \rightarrow \operatorname{inv}\left(E_{\mathrm{T}}^{\text {miss }}\right)[30], W / Z(\rightarrow \mathrm{jj}) \mathrm{h} \rightarrow \operatorname{inv}\left(E_{\mathrm{T}}^{\text {miss }}\right)[31], \mathrm{VBF}(\rightarrow \mathrm{jj}) \mathrm{h} \rightarrow$ $\operatorname{inv}\left(E_{\mathrm{T}}^{\mathrm{miss}}\right)[32]$, where the first analysis contains the $7 \mathrm{TeV}$ data.

Table 4 shows upper limits on the $\mathrm{BR}(h \rightarrow$ inv $)$ at $95 \%$ CL and other CL for different analyses and their statistical combination. The combination of the invisible channels only resulted in $\mathrm{BR}(h \rightarrow i n v)<0.25$ obs $(0.27 \exp )$.

Table 4: Summary of upper bounds on $\operatorname{BR}(h \rightarrow$ inv) [27].

\begin{tabular}{c|cccccc}
\hline Results & Observed & $-2 \sigma$ & $-1 \sigma$ & Expected & $+1 \sigma$ & $+2 \sigma$ \\
\hline VBF $h$ & 0.28 & 0.16 & 0.21 & 0.31 & 0.41 & 0.56 \\
$Z(\rightarrow \ell \ell) h$ & 0.75 & 0.33 & 0.45 & 0.62 & 0.86 & 1.19 \\
$V(\rightarrow j j) h$ & 0.78 & 0.46 & 0.62 & 0.86 & 1.19 & 1.60 \\
\hline Combined Results & 0.25 & 0.13 & 0.18 & 0.27 & 0.35 & 0.47 \\
\hline
\end{tabular}

Combined result was then combined statistically with the measured visible decay rates of the Higgs boson. A physical boundary of $B R_{i n v}>0$ was required. The most general result uses independent coupling parameters $\kappa_{W}, \kappa_{Z}, \kappa_{t}, \kappa_{b}, \kappa_{\tau}, \kappa_{\mu}, \kappa_{g}, \kappa_{\gamma}, \kappa_{Z \gamma}$ and $\mathrm{BR}_{\text {inv }}$. Upper limit of $0.23(0.24)$ obs(exp.) were set at $95 \%$ CL. The likelihood scan for that statistical fit is shown in Fig. 8.

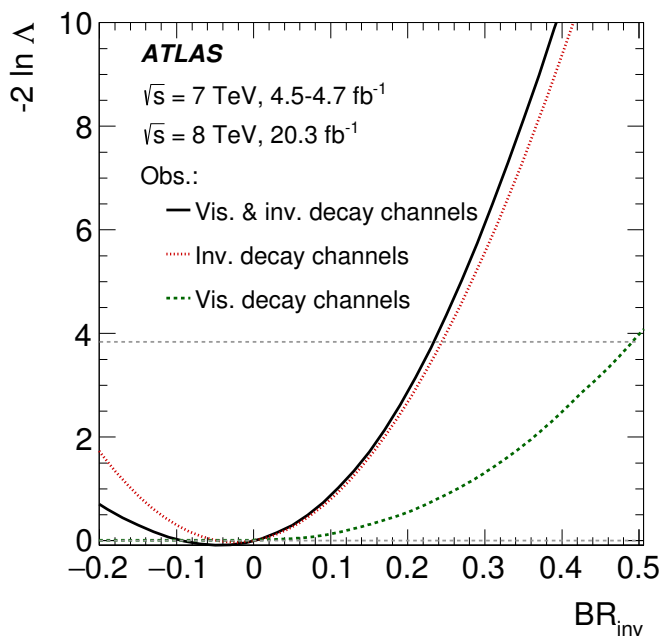

Figure 8. Likelihood scans of the Higgs' invisible branching ratio [27].

\subsection{Higgs Portal Interpretation}

The $90 \%$ CL upper limit of $0.22(0.23)$ obs $(\exp )$ on $B R(H \rightarrow i n v)$ was used to constrain on a Higgs-portal dark matter scenario [29], where the Higgs boson is the only mediator for interactions between WIMPs and other SM particles. It is sensitive for WIMP's mass < $m_{h} / 2$. Limits are set on the cross section between WIMPs and nucleons as a function of the 
WIMP mass, as shown in Fig. 9. The form factor (Higgs-nucleon coupling) $f_{N}$ is taken to be $0.33_{-0.07}^{+0.30}[29]$.

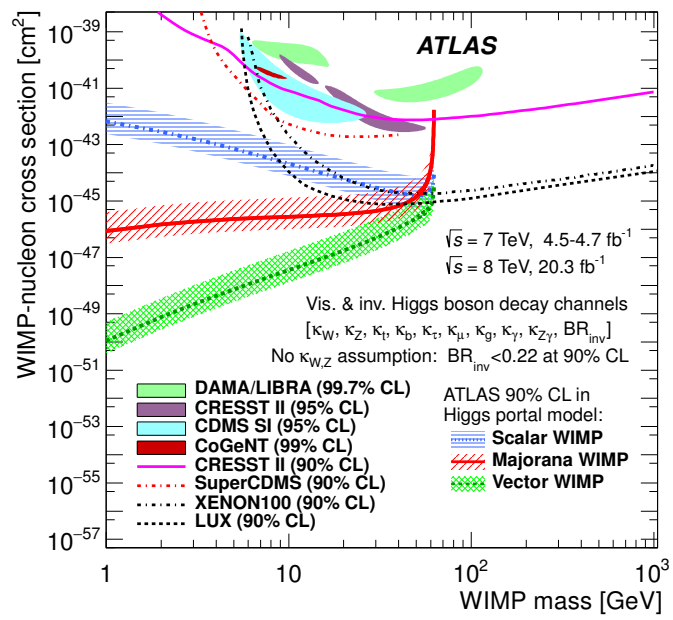

Figure 9. Upper limit at $90 \% \mathrm{CL}$ on the WIMP-nucleon scattering cross section, ATLAS's compared with the direct searches [27].

\section{Conclusion}

The ATLAS collaboration has performed many searches for BSM Higgs bosons using the $7 \mathrm{TeV}$ and $8 \mathrm{TeV}$ datasets: high mass Higgs search, LFV Higgs decay, Higgs decays to pseudoscalar particles in the context of NMSSM, Higgs decays to four photons via pseudoscalar particles, Higgs decay into invisible particles. No significant excess for BSM Higgs physics has been observed yet. Precise measurements of the Higgs boson couplings allow to constrain new phenomena: mass scaling ( $\epsilon: 0.018 \pm 0.039, M: 224+14-12 \mathrm{GeV}$ ), Minimal Composite Higgs models $(f>710(780) \mathrm{GeV}$ MCHM4(5)), Two Higgs Doublet Models (Alignment limit within $1 \sigma)$, Higgs to invisible decays $\left(B R_{\text {inv }}<0.23\right)$ and subsequent constraint on Higgsportal dark matter. Much better results with the new data from the $\sqrt{s}=13 \mathrm{TeV}$ run at the LHC are expected.

\section{References}

[1] ATLAS Collaboration, Eur. Phys. J. C75, 476 (2015), 1506.05669

[2] CMS Collaboration (CMS), Phys. Rev. D92, 012004 (2015), 1411.3441

[3] ATLAS and CMS Collaborations, Phys. Rev. Lett. 114, 191803 (2015), 1503.07589

[4] ATLAS Collaboration (2015), 1507.04548

[5] CMS Collaboration, Eur. Phys. J. C75, 212 (2015), 1412.8662

[6] LHC Higgs cross section working group, S. Dittmaier, C. Mariotti, G. Passarino, S. Heinemeyer, R. Tanaka (Eds.) (2013), 1307.1347

[7] T. Lee, Phys. Rev. D. 8, 1226 (1973)

[8] J.F. Gunion, H.E. Haber, Phys. Rev. D. 67, 075019 (2003), hep-ph/0207010

[9] G. Branco et al., Phys. Rept. 516, 1 (2012), 1106.0034

[10] U. Ellwanger, J.F. Gunion, C. Hugonie (2001), pp. 178-188, hep-ph/0111179 
[11] D. Curtin et al., Phys. Rev. D90, 075004 (2014), 1312.4992

[12] K. Agashe, R. Contino, A. Pomarol, Nucl. Phys. B 719, 165 (2005), hep-ph/0412089

[13] R. Contino, L. Da Rold, A. Pomarol, Phys. Rev. D 75, 055014 (2007)

[14] D.C. et al., Astrophys. J. 648, L109 (2006), astro-ph/0608407

[15] I. Antoniadis, M. Tuckmantel, F. Zwirner, Nucl.Phys. B707, 215 (2005), hep-ph/0410165

[16] N. Arkani-Hamed, S. Dimopoulos, G. Dvali, J. March-Russell, Phys.Rev. D65, 024032 (2002), hep-ph/9811448

[17] A. Datta, K. Huitu, J. Laamanen, B. Mukhopadhyaya, Phys.Rev. D70, 075003 (2004), hep-ph/0404056

[18] S. Kanemura, S. Matsumoto, T. Nabeshima, N. Okada, Phys.Rev. D82, 055026 (2010), 1005.5651

[19] A. Djouadi, A. Falkowski, Y. Mambrini, J. Quevillon, Eur.Phys.J. C73, 2455 (2013), 1205.3169

[20] ATLAS Collaboration, JINST 3, S08003 (2008)

[21] ATLAS Collaboration (2015), 1509.00389

[22] ATLAS Collaboration (2015), 1507.05930

[23] R. Harnik, J. Kopp, J. Zupan, JHEP 03, 026 (2013), 1209.1397

[24] ATLAS Collaboration (2015), 1508.03372

[25] ATLAS Collaboration, Phys. Rev. D92, 052002 (2015), 1505.01609

[26] ATLAS Collaboration (2015), 1509.05051

[27] ATLAS Collaboration (2015), 1509.00672

[28] J. Ellis, T. You, JHEP 06, 103 (2013), 1303.3879

[29] A. Djouadi, O. Lebedev, Y. Mambrini, J. Quevillon, Phys.Lett. B709, 65 (2012), 1112. 3299

[30] ATLAS Collaboration (ATLAS), Phys. Rev. Lett. 112, 201802 (2014), 1402.3244

[31] ATLAS Collaboration (ATLAS), Eur. Phys. J. C75, 337 (2015), 1504.04324

[32] ATLAS Collaboration (2015), 1508.07869 